Omni-Akuatika, 16 (2): 151 - 166, 2020
ISSN: 1858-3873 print / 2476-9347 online
Research Article
journal homepage: http://ojs.omniakuatika.net

\title{
Spatio-Temporal Assessment of Zooplankton of Uta Ewa Estuary, Akwa Ibom State, Nigeria
}

\author{
Udeme Effiong Jonah*, Emeka Donald Anyanwu, Diane Akudo Avoaja \\ Department of Zoology and Environmental Biology, Michael Okpara University of Agriculture, Umudike, Abia \\ State, Nigeria \\ ${ }^{*}$ Corresponding author: udemejonah@gmail.com
}

Received 24 September 2020; Accepted 30 November 2020; Available online 31 December 2020

\begin{abstract}
Estuaries are influenced by the mixture of river water with seawater; creating unique ecosystems with several physical and chemical processes affecting the water quality. Spatial and temporal assessment of the composition, abundance, and distribution of zooplankton fauna of Uta Ewa Estuarine water system was carried out between May 2019 and February 2020 to assess the effects of anthropogenic activities on the zooplankton assemblage. Water and Zooplankton samples were collected from three (3) stations using standard procedures. Some parameters like water temperature, dissolved oxygen, hydrogen-ion, electrical conductivity, and turbidity were determined in-situ. The ranges of the physico-chemical parameters were: water temperature $\left(24.9-25.3^{\circ} \mathrm{C}\right), \mathrm{EC}(62.3-70.9 \mathrm{mS} / \mathrm{m}), \mathrm{pH}(6.5-6.7)$, turbidity (12.028.0NTU), DO (3.8-4.7mg/L), BOD (2.3-3.2mg/L), phosphate $(3.2-5.2 \mathrm{mg} / \mathrm{L})$, and nitrate $(3.0-6.3 \mathrm{mg} / \mathrm{L})$. ANOVA showed a significant difference $(p<0.05)$ in the spatial and temporal means values of some parameters. A total of 1,067 individuals from 30 zooplankton taxa and 4 taxonomic groups were recorded. Rotifers $(33.4 \%)$ were the dominant group, followed by protozoa $(32.9 \%)$, copepods $(20.9 \%)$ and cladocerans (13.8\%). Station 1 had the highest abundance (388 individuals), station 2 (303 individuals) and station 3(375 individuals). The higher number of individuals (193) was recorded in August 2019. The biodiversity indices pointed to slightly polluted to stable environment. This study showed that the water quality and zooplankton community was influenced by anthropogenic activities both spatially and temporally but season also played a major role in the temporal variation. In conclusion, the water quality was deteriorating due to anthropogenic activities, which in turn affected the structure of zooplankton community.
\end{abstract}

Keywords: Abundance, Assessment, Zooplankton, Physicochemical, Estuary

\begin{abstract}
Abstrak
Estuari dipengaruhi oleh campuran air sungai dengan air laut; menciptakan ekosistem yang unik dengan beberapa proses fisik dan kimiawi yang mempengaruhi kualitas air. Penilaian spasial dan temporal komposisi, kelimpahan, dan sebaran fauna zooplankton sistem perairan Estuari Uta Ewa dilakukan antara Mei 2019 dan Februari 2020 untuk menilai pengaruh aktivitas antropogenik terhadap kumpulan zooplankton. Sampel air dan Zooplankton dikumpulkan dari tiga (3) stasiun dengan menggunakan prosedur standar. Beberapa parameter seperti suhu air, oksigen terlarut, ion hidrogen, konduktivitas listrik, dan kekeruhan ditentukan secara in-situ. Kisaran parameter fisika-kimiawi adalah: suhu air (24,9$\left.25,3^{\circ} \mathrm{C}\right)$, EC (62,3-70,9ms / m), pH (6,5-6,7), kekeruhan (12,0-28,0NTU), DO (3,8-4,7mg / L), BOD (2.3$3.2 \mathrm{mg} / \mathrm{L})$, fosfat $(3.2-5.2 \mathrm{mg} / \mathrm{L})$, dan nitrat $(3.0-6.3 \mathrm{mg} / \mathrm{L})$. ANOVA menunjukkan perbedaan yang signifikan $(p<0,05)$ pada nilai rata-rata spasial dan temporal dari beberapa parameter. Sebanyak 1.067 individu dari 30 zooplankton taksa dan 4 kelompok taksonomi dicatat. Rotifer $(33,4 \%)$ merupakan kelompok dominan, diikuti oleh protozoa (32,9\%), copepoda $(20,9 \%)$ dan cladocerans $(13,8 \%)$. Stasiun 1 memiliki kelimpahan tertinggi (388 individu), stasiun 2 (303 individu) dan stasiun 3 (375 individu). Jumlah individu yang lebih tinggi (193) tercatat pada Agustus 2019. Indeks keanekaragaman hayati menunjukkan sedikit tercemar hingga lingkungan stabil. Studi ini menunjukkan bahwa kualitas air dan komunitas zooplankton dipengaruhi oleh aktivitas a ntropogenik baik secara spasial maupun temporal
\end{abstract}


tetapi musim juga berperan besar dalam variasi temporal. Sebagai kesimpulan, kualitas air menurun akibat aktivitas antropogenik yang pada akhirnya mempengaruhi struktur komunitas zooplankton.

Kata Kunci: Kelimpahan, Pengkajian, Zooplankton, Fisikokimia, Muara

\section{Introduction}

Complex and dynamic aquatic ecosystems exist within the estuary and coastal areas (Morris et al., 1995). The mixture of river water with seawater in the estuary involves several physical and chemical processes which may affect the water quality (Anitha \& Kumar, 2013). Zooplankton are microscopic animals that are an indispensable constituent of aquatic food webs; an important link in the energy conversion from producers to consumers (Sousa et al., 2008; Sharma et al., 2010). They could be non-motile or very poor swimmers; drifting in the water column of water bodies and depends on water to get to any great distance (Ferdous \& Muktadir, 2009). They are closely linked to the environment, quickly responding to changes and their distribution is strongly influenced by environmental parameters (Roman et al., 2012; Vincent et al., 2012; Lin et al., 2014; Ergönül et al., 2016). Zooplanktons have been reported to be good bioindicators of water quality (Ferdous \& Muktadir, 2009; Davis \& Ugwumba, 2013; Dorak, 2013; Kutama et al., 2014 and Job et al., 2017). Nutrients availability, the stability of the environment, space and time are major factors determining the variations in zooplankton density (Kar et al., 2018). Related studies have shown that zooplankton abundance in the water body is associated with the seasonal fluctuations in some aspect of hydrochemistry concentration in the water body (Essien-lbok \& Ekpo, 2015; Jonah \& George, 2019). Human activities such as industrial, indiscriminate disposal of wastes and agriculture are leading contributors to pollution in the aquatic ecosystem (Anyanwu et al., 2013; Jonah et al., 2019; Anyanwu \& Umeham 2020a; Jonah et al., 2020); these activities alter the favorable water quality for zooplankton growth and development. Uta Ewa Estuary is characterized by heterogeneous forms of anthropogenic activities, and it is the major source of water for irrigation and other domestic purposes communities within its watershed. In view of the level of anthropogenic activities in the estuary, Akpan \& Etim (2015) studied the seasonal and spatial variations in water quality and ecological adversity of the Estuary while Esenowo et al. (2016), Egwali et al. (2018) and Akpan et al. (2019) studied aspects of shellfish (Decapoda: Crustacea) in the Estuary but there is no study on the zooplankton. Hence, the objective of this study was to assess the effect of anthropogenic activities and season on the zooplankton assemblage of the estuary.

\section{Material and methods}

\subsection{Study Area and Sampling Stations}

Uta Ewa Estuary is located in Ikot Abasi Local Government Area, Akwa Ibom State, Nigeria, within Latitude 4'32'49" North and Longitude $7^{\circ} 30^{\prime} 13^{\prime \prime}$ East (Fig.1). The estuary lies on the Western bank of Enong Greek, about 12 $\mathrm{km}$ from the mouth of Imo River (Akpan et al., 2019), drain into Atlantic Ocean. The study area is about 11.6 meter deep; characterized by a tropical climate of wet and dry season period. The brackish water is a fishing ground for several near-shore fishing settlements as well as the indigenous community. The common mangrove plants observed were Rhizophora raceinosa, Nypa fruticans and Avicannia africana. The anthropogenic activities observed include fishing, farming, boat building, logging, laundering, selling of food-stuff (miscellaneous items) and transportation of goods. The estuary receives wastes from nearby settlements, markets, and from domestic activities. Three sampling stations were selected along the study stretch of the water body, based on accessibility and the nature of anthropogenic activities. Station 1 is upstream with minimal anthropogenic activities. The water is clear with high velocity. The substrate is sandy. The vegetation around here is dominated by mangrove plants like Nypa palm (Nypa fruticans), Rhizophora racemosa, and other aquatic plants. Station 2 is located $3 \mathrm{~km}$ downstream of station 1 and close to residential settlements and local markets. The substrate is mixed. The human activities observed include bathing, cutting of mangrove plants for firewood, fishing, laundry and other domestic activities. Station 3 is $2 \mathrm{~km}$ downstream of station 2. It is dominated by Rhizophora species along the shores; observed human activities include fishing, laundry, bathing, cutting of mangrove plants for firewood and other domestic activities. The station received waste from various anthropogenic activities and from nearby settlements. The Substrate is also sandy. 


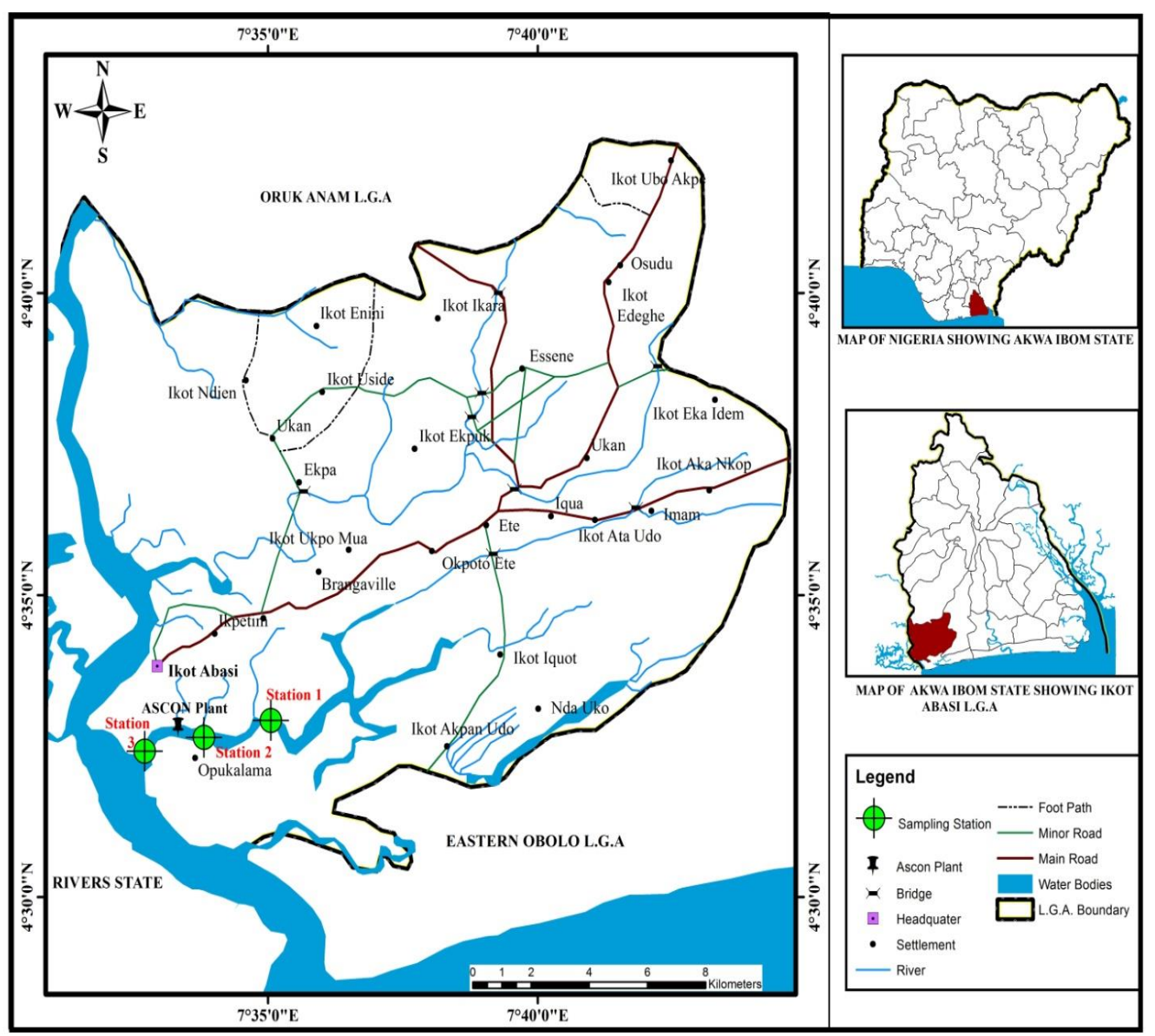

Figure 1. Map of Ikot Abasi Local Government Area, Akwa Ibom State, Nigeria showing Uta Ewa Estuary and the sampling Stations.

\subsection{Samples Collection and Analysis}

Water Samples: The water sampling was carried out monthly between May 2019, and February 2020. One liter water sampler was used and transferred into a clean one liter sterilized plastic bottles. Water temperature, dissolved oxygen, hydrogen-ion concentration, electrical conductivity, total dissolved solids, and turbidity were determined in-situ using Extech meter probes. Other parameters were determined in the laboratory using standard methods (AOAC, 2000; APHA, 2005). One-way ANOVA was used for statistical analysis of the data for significant differences. The source of significant differences between means was determined by Tukey Pairwise test. All statistical analysis was performed with PAST software package (Version 3.24) (Hammer et al., 2001).

Zooplankton Samples: Zooplankton samples were collected using a standard plankton net of $55 \mu \mathrm{m}$ mesh size. The plankton net was towed horizontally for five (5) minutes on the water body by a boat at a regulated velocity; the concentrated samples were pure into $250 \mathrm{ml}$ of sampling bottles and fixed with two drops of Lugol's iodine solution. The samples were transported to the laboratory for analysis. In the laboratory, one $\mathrm{ml}$ of the preserved sample was taken as a sub- sample using a pipette. The collected sample was put on the Sedgwick-rafter counting chamber and viewed under a light binocular microscope (Nikon 400 binocular microscope) using a low magnification of $x 10$. Zooplanktons were sorted into different groups and the cells per $\mathrm{ml}$ were counted (Schoen 1988). Identification work was done using key literature by Jeje and Fernando (1986); Jeje (1988); Boxshall \& Braide (1991) and Dang et al. (2015). The identification was made to the lowest practicable taxonomic level. The community structure of the zooplankton was determined using ecological indices (Shannon-Weiner index $(\mathrm{H})$, Margalef index (d), and Evenness index). 


\section{Results}

\section{1. Physico-chemical parameters}

The spatial variation of the physicochemical parameters are summarized in Table 1 and the temporal variation in Table 2 . The water temperature values ranged from $22.8^{\circ} \mathrm{C}$ to $28.0^{\circ} \mathrm{C}$. The lowest value was recorded in July 2019 in station 1 while the highest was recorded in January 2020 in station 2. There was no significant difference $(P>0.05)$ in the stations when one-way ANOVA was applied while there was a significant difference $(P<0.05)$ in the months. January 2020 was significantly higher than the other months, followed by OctoberDecember 2019. The highest mean values were $25.3^{\circ} \mathrm{C}$ (station 2) and $27.8^{\circ} \mathrm{C}$ (January 2020).

Electrical conductivity (EC) spatially ranged between $45.0 \mathrm{mS} / \mathrm{m}$ and $88.0 \mathrm{mS} / \mathrm{m}$. The lowest value was recorded in October 2019 in station 1 while the highest values were recorded in May and September 2019 in station 3. There was no significant difference in both spatial and temporal values $(P>0.05)$. The highest mean values were $70.9 \mathrm{mS} / \mathrm{m}$ (station 3) and $77.0 \mathrm{mS} / \mathrm{m}$ (May 2019).

The $\mathrm{pH}$ was moderate acidic to alkaline, ranging from 6.2-7.6. The lowest was recorded in station 3 (June 2019) while the highest was recorded in station 2 (October 2019). There was no significant difference in both spatial and temporal values $(P>0.05)$. The highest mean values were 6.7 (station 2) and 7.0 (October 2019).

The turbidity values ranged between 3.2 and 45.1 NTU. The lowest was recorded in station 1 (January 2020) while the highest was recorded in station 2 (August 2019). Stations 2 and 3 were significantly different $(P>0.05)$ from station 1 while August 2019 was significantly different $(P<0.05)$ from November 2019 to February 2020. The highest mean values were 28.2 NTU (station 2) and 33.4 NTU (August 2019).

Dissolved Oxygen (DO) ranged from 1.9 to $7.8 \mathrm{mg} / \mathrm{l}$. The lowest value was recorded in station 3 (August 2019) while the highest was recorded in station 1 (December 2019). Stations 2 and 3 were significantly $(P<0.05)$ lower than station 1 while December 2019 was significantly $(P<0.05)$ higher than May to September 2019. The highest mean values were $4.7 \mathrm{mg} / \mathrm{l}$ (station 1) and $6.6 \mathrm{mg} / \mathrm{l}$ (December 2019).

Biochemical Oxygen Demand (BOD) values ranged between 0.2 and $5.3 \mathrm{mg} / \mathrm{l}$. The lowest value was recorded in station (January 2020) while the highest was recorded in station 2 (June 2019). There was no significant difference $(P>0.05)$ in the stations but June 2019 was significantly different $(P<0.05)$ from January 2020.The highest mean values were $3.2 \mathrm{mg} / \mathrm{l}$ (station 2) and $4.0 \mathrm{mg} / \mathrm{l}$ (June 2019).

Table 1. Spatial variation of physico-chemical parameters of Uta Ewa Estuary Water

\begin{tabular}{lllll}
\hline Parameter & \multicolumn{1}{c}{$\begin{array}{c}\text { Station } \mathbf{X} \pm \text { SEM } \\
\text { Temp }\left({ }^{\circ} \mathrm{C}\right)\end{array}$} & \multicolumn{1}{c}{$\begin{array}{c}\text { Station 2 } \\
\mathbf{X} \pm \text { SEM }\end{array}$} & $\begin{array}{c}\text { Station 3 } \\
\mathbf{X} \pm \text { SEM }\end{array}$ & P-value \\
EC $(\mathrm{mS} / \mathrm{m})$ & $25.1 \pm 0.54$ & $25.3 \pm 0.55$ & $24.9 \pm 0.57$ & $\mathrm{P}>0.05$ \\
& $(22.8-27.5)$ & $(23.0-28.0)$ & $(22.9-27.9)$ & \\
$\mathrm{pH}$ & $63.8 \pm 2.99$ & $62.3 \pm 3.29$ & $70.9 \pm 4.00$ & $\mathrm{P}>0.05$ \\
& $(45.0-75.0)$ & $(49.0-75.0)$ & $(53.0-88.0)$ & \\
Turb $(\mathrm{NTU})$ & $6.6 \pm 0.12$ & $6.7 \pm 0.15$ & $6.5 \pm 0.05$ & $\mathrm{P}>0.05$ \\
& $(6.3-7.5)$ & $(6.3-7.6)$ & $(6.2-6.7)$ & \\
DO $(\mathrm{mg} / \mathrm{l})$ & $12.0 \pm 2.66^{\mathrm{a}}$ & $28.2 \pm 2.37^{\mathrm{b}}$ & $21.8 \pm 2.55^{\mathrm{b}}$ & $\mathrm{P}<0.05$ \\
& $(3.2-30.4)$ & $(18.9-45.1)$ & $(12.0-35.5)$ & \\
BOD $(\mathrm{mg} / \mathrm{l})$ & $4.7 \pm 0.46^{\mathrm{a}}$ & $3.8 \pm 0.42^{\mathrm{b}}$ & $3.9 \pm 0.49^{\mathrm{b}}$ & $\mathrm{P}<0.05$ \\
& $(2.9-7.8)$ & $(2.2-6.0)$ & $(1.9-6.8)$ & \\
Phosphate $(\mathrm{mg} / \mathrm{l})$ & $2.3 \pm 0.33$ & $3.2 \pm 0.39$ & $3.0 \pm 0.43$ & $\mathrm{P}>0.05$ \\
& $(0.2-3.6)$ & $(1.7-5.3)$ & $(1.0-4.6)$ & \\
Nitrate $(\mathrm{mg} / \mathrm{l})$ & $3.2 \pm 0.40^{\mathrm{a}}$ & $4.8 \pm 0.55^{\mathrm{b}}$ & $5.2 \pm 0.54^{\mathrm{b}}$ & $\mathrm{P}<0.05$ \\
& $(1.5-5.4)$ & $(2.3-7.7)$ & $(2.8-7.8)$ & \\
& $3.0 \pm 0.55^{\mathrm{a}}$ & $6.3 \pm 0.34^{\mathrm{b}}$ & $5.5 \pm 0.55^{\mathrm{b}}$ & $\mathrm{P}<0.05$ \\
\hline
\end{tabular}

$a, b=$ means with different superscripts across the row are significantly different at $\mathrm{P}<0.05$; $\mathrm{SEM}=\mathrm{Standard}$ Error of mean. 
Table 2. Temporal variation of physico-chemical parameters of Uta Ewa Estuary Water

\begin{tabular}{|c|c|c|c|c|c|c|c|c|}
\hline $\begin{array}{c}\text { Months/ } \\
\text { Parameters }\end{array}$ & $\begin{array}{c}\text { Temp } \\
(\text { oC) } \\
\text { X } \pm \text { SEM } \\
\end{array}$ & $\begin{array}{c}\mathrm{EC}(\mathrm{mS} / \mathrm{m}) \\
\mathrm{X} \pm \mathrm{SEM}\end{array}$ & $\begin{array}{c}\mathrm{pH} \\
\mathrm{X} \pm \mathrm{SEM}\end{array}$ & $\begin{array}{c}\text { Turb } \\
\text { (NTU) } \\
\text { X } \pm \text { SEM } \\
\end{array}$ & $\begin{array}{c}\mathrm{DO}(\mathrm{mg} / \mathrm{l}) \\
\mathrm{X} \pm \mathrm{SEM}\end{array}$ & $\begin{array}{l}\text { BOD } \\
(\mathrm{mg} / \mathrm{l}) \\
\mathrm{X} \pm \text { SEM }\end{array}$ & $\begin{array}{c}\text { Phosphate } \\
\text { (mg/l) } \\
\text { X } \pm \text { SEM } \\
\end{array}$ & $\begin{array}{c}\text { Nitrate } \\
(\mathrm{mg} / \mathrm{l}) \\
\mathrm{X} \pm \mathrm{SEM} \\
\end{array}$ \\
\hline May 2019 & $\begin{array}{c}23.6 \pm 0.32^{\mathrm{ab}} \\
(23.0-24.0)\end{array}$ & $\begin{array}{l}77.0 \pm 5.86 \\
(68.0- \\
88.0)\end{array}$ & $\begin{array}{c}6.5 \pm 0.09 \\
(6.3-6.6)\end{array}$ & $\begin{array}{c}21.4 \pm 4.92^{\mathrm{ab}} \\
(15.1- \\
31.1)\end{array}$ & $\begin{array}{l}2.8 \pm 0.29^{a} \\
(2.3-3.3)\end{array}$ & $\begin{array}{l}3.5 \pm 0.81^{1 b c} \\
(2.0-4.8)\end{array}$ & $\begin{array}{c}4.3 \pm 0.38^{\mathrm{abc}} \\
(3.5-4.7)\end{array}$ & $\begin{array}{l}6.0 \pm 0.75^{\mathrm{ab}} \\
(4.7-7.3)\end{array}$ \\
\hline Jun. 2019 & $\begin{array}{c}23.0 \pm 0.06^{\mathrm{a}} \\
(22.9-23.1)\end{array}$ & $\begin{array}{l}71.3 \pm 1.86 \\
(69.0- \\
75.0)\end{array}$ & $\begin{array}{c}6.4 \pm 0.12 \\
(6.2-6.6)\end{array}$ & $\begin{array}{c}24.6 \pm 3.18^{\mathrm{ab}} \\
(21.0- \\
30.9)\end{array}$ & $\begin{array}{l}3.2 \pm 0.50^{\mathrm{ac}} \\
(2.2-3.9)\end{array}$ & $\begin{array}{l}4.0 \pm 0.64^{b} \\
(3.2-5.3)\end{array}$ & $\begin{array}{l}5.9 \pm 1.23^{b c} \\
(3.6-7.8)\end{array}$ & $\begin{array}{l}6.0 \pm 1.70^{\mathrm{ab}} \\
(2.6-8.0)\end{array}$ \\
\hline Jul. 2019 & $\begin{array}{c}22.9 \pm 0.09^{\mathrm{a}} \\
(22.8-23.1)\end{array}$ & $\begin{array}{l}71.0 \pm 3.51 \\
(64.0- \\
75.0)\end{array}$ & $\begin{array}{c}6.4 \pm 0.03 \\
(6.3-6.4)\end{array}$ & $\begin{array}{l}25.6 \pm 8.69^{a b} \\
(8.3-35.5)\end{array}$ & $\begin{array}{l}3.6 \pm 0.38^{\mathrm{ac}} \\
(2.9-4.2)\end{array}$ & $\begin{array}{l}3.9 \pm 0.38^{b c} \\
(3.3-4.6)\end{array}$ & $\begin{array}{l}4.0 \pm 1.39^{a} \\
(2.5-6.8)\end{array}$ & $\begin{array}{l}5.0 \pm 1.54^{\mathrm{ab}} \\
(2.5-7.8)\end{array}$ \\
\hline Aug. 2019 & $\begin{array}{c}24.6 \pm 0.31^{\mathrm{b}} \\
(24.0-25.0)\end{array}$ & $\begin{array}{l}72.3 \pm 4.84 \\
(67.0- \\
82.0)\end{array}$ & $\begin{array}{c}6.6 \pm 0.09 \\
(6.4-6.7)\end{array}$ & $\begin{array}{c}33.4 \pm 6.05^{\mathrm{b}} \\
(24.8- \\
45.1)\end{array}$ & $\begin{array}{l}2.4 \pm 0.29^{a} \\
(1.9-2.9)\end{array}$ & $\begin{array}{l}3.8 \pm 0.39^{b c} \\
(3.4-4.6)\end{array}$ & $\begin{array}{l}6.9 \pm 0.74^{b} \\
(5.4-7.7)\end{array}$ & $\begin{array}{l}5.8 \pm 0.06^{\mathrm{ab}} \\
(5.7-5.9)\end{array}$ \\
\hline Sep. 2019 & $\begin{array}{l}24.0 \pm 0.09^{\mathrm{ab}} \\
(23.8-24.1)\end{array}$ & $\begin{array}{l}72.0 \pm 8.32 \\
(60.0- \\
88.0)\end{array}$ & $\begin{array}{c}6.4 \pm 0.07 \\
(6.3-6.5)\end{array}$ & $\begin{array}{c}24.7 \pm 6.58^{\mathrm{ab}} \\
(12.0- \\
34.0)\end{array}$ & $\begin{array}{l}3.4 \pm 0.57^{\mathrm{ac}} \\
(2.3-4.1)\end{array}$ & $\begin{array}{l}3.6 \pm 0.03^{b c} \\
(3.5-3.6)\end{array}$ & $\begin{array}{l}5.7 \pm 0.61^{b c} \\
(4.8-6.9)\end{array}$ & $\begin{array}{l}6.3 \pm 0.98^{b} \\
(4.6-8.0)\end{array}$ \\
\hline Oct. 2019 & $\begin{array}{c}26.3 \pm 0.27^{\circ} \\
(25.9-26.8)\end{array}$ & $\begin{array}{l}56.0 \pm 8.62 \\
(45.0- \\
73.0)\end{array}$ & $\begin{array}{c}7.0 \pm 0.32 \\
(6.5-7.6)\end{array}$ & $\begin{array}{l}19.0 \pm 4.75^{\mathrm{ab}} \\
(9.6-25.0)\end{array}$ & $\begin{array}{l}4.9 \pm 0.17^{b c} \\
(4.7-5.2)\end{array}$ & $\begin{array}{l}2.6 \pm 0.38^{b c} \\
(2.2-3.4)\end{array}$ & $\begin{array}{l}4.0 \pm 0.58^{\mathrm{ac}} \\
(3.4-5.2)\end{array}$ & $\begin{array}{l}5.6 \pm 0.64^{\mathrm{ab}} \\
(4.4-6.6)\end{array}$ \\
\hline Dec. 2019 & $\begin{array}{c}25.9 \pm 0.09^{c} \\
(25.7-26.0) \\
26.0 \pm 0.58^{c} \\
(25.0-27.0)\end{array}$ & $\begin{array}{l}59.7 \pm 6.06 \\
(49.0-70 .) \\
59.0 \pm 3.79 \\
(53.0- \\
66.0)\end{array}$ & $\begin{array}{c}6.5 \pm 0.03 \\
(6.4-6.5) \\
6.4 \pm 0.06 \\
(6.3-6.5)\end{array}$ & $\begin{array}{c}15.6 \pm 5.52^{\mathrm{a}} \\
(8.3-26.4) \\
16.0 \pm 4.40^{\mathrm{a}} \\
(7.3-21.6)\end{array}$ & $\begin{array}{c}5.4 \pm 0.50^{\mathrm{bc}} \\
(4.4-6.0) \\
6.6 \pm 0.76^{\mathrm{b}} \\
(5.2-7.8)\end{array}$ & $\begin{array}{l}1.7 \pm 0.38^{b c} \\
(1.0-2.3) \\
1.6 \pm 0.28^{a c} \\
(1.0-1.9)\end{array}$ & $\begin{array}{c}4.3 \pm 0.39 \mathrm{abc} \\
(3.5-4.8) \\
3.3 \pm 0.56^{\mathrm{ac}} \\
(2.2-4.0)\end{array}$ & $\begin{array}{l}4.5 \pm 1.73^{\mathrm{ab}} \\
(1.0-6.3) \\
4.2 \pm 1.02^{\mathrm{ab}} \\
(2.3-5.8)\end{array}$ \\
\hline Jan. 2020 & $\begin{array}{c}27.8 \pm 0.15^{d} \\
(27.5-28.0)\end{array}$ & $\begin{array}{l}57.9 \pm 3.94 \\
(53.0- \\
65.7)\end{array}$ & $\begin{array}{c}6.9 \pm 0.33 \\
(6.4-7.5)\end{array}$ & $\begin{array}{l}12.0 \pm 4.62^{\mathrm{a}} \\
(3.2-18.9)\end{array}$ & $\begin{array}{l}4.8 \pm 0.45^{\mathrm{ab}} \\
(4.2-5.7)\end{array}$ & $\begin{array}{l}1.3 \pm 0.61^{\mathrm{a}} \\
(0.2-2.3)\end{array}$ & $\begin{array}{l}3.4 \pm 1.00^{\mathrm{ac}} \\
(1.5-4.9)\end{array}$ & $\begin{array}{l}3.3 \pm 1.23^{\mathrm{ab}} \\
(1.4-5.6)\end{array}$ \\
\hline Feb. 2020 & $\begin{array}{l}27.0 \pm 0.12^{\mathrm{cd}} \\
(26.8-27.2)\end{array}$ & $\begin{array}{l}60.3 \pm 7.42 \\
51.0- \\
75.0)\end{array}$ & $\begin{array}{c}6.9 \pm 0.33 \\
(6.5-7.5)\end{array}$ & $\begin{array}{c}14.7 \pm 5.83^{\mathrm{a}} \\
(4.4-24.6)\end{array}$ & $\begin{array}{l}4.4 \pm 0.76^{\mathrm{ab}} \\
(3.2-5.8)\end{array}$ & $\begin{array}{l}2.5 \pm 0.53^{b c} \\
(1.5-3.3)\end{array}$ & $\begin{array}{c}2.3 \pm 0.29^{a} \\
(1.8-2.8)\end{array}$ & $\begin{array}{c}2.8 \pm 1.04^{a} \\
1.0-4.6\end{array}$ \\
\hline P-value & $\mathrm{P}<0.05$ & $P>0.05$ & $P>0.05$ & $P<0.05$ & $\mathrm{P}<0.05$ & $P<0.05$ & $\mathrm{P}<0.05$ & $\mathrm{P}<0.05$ \\
\hline
\end{tabular}

$a, b=$ means with different superscripts across the row are significantly different at $P<0.05$; SEM $=$ Standard Error of mean.

The values for phosphate ranged between 1.5 and $7.8 \mathrm{mg} / \mathrm{l}$. The lowest value was recorded in station 1 (January 2020) while the highest value was recorded in station 3 (June 2019). Station 2 and 3 were significantly $(P<0.05)$ higher than station 1 and August 2019 was significantly different $(P<0.05)$ different from July 2019, October 2019 to February 2020.

The highest mean values were $5.2 \mathrm{mg} / \mathrm{l}$ (station 2) and $6.9 \mathrm{mg} / \mathrm{l}$ (August 2019). The values for nitrate ranged between 1.0 and 8.0 $\mathrm{mg} / \mathrm{l}$. The lowest value was recorded in station 1 (November 2019 and February 2020) while the highest value was recorded in stations 2 (June 2019) and 3 (September 2019). Station 2 and 3 were also significantly $(P<0.05)$ higher than station 1 while September 2019 was significantly different $(P<0.05)$ different from February 2020. The highest mean values were $6.3 \mathrm{mg} / \mathrm{l}$ in station 2 and August 2019 for spatial and temporal respectively.

\section{2. Zooplankton Composition, Abundance, and Distribution}

The spatial and temporal zooplankton species compositions are presented in Tables 3 and 4. This study recorded a total of 1,067 zooplankton individuals, comprising of four (4) taxonomic groups and thirty (30) taxa. Rotifers contributed $33.4 \%$ of the zooplankton population with 356 individuals from ten (10) taxa, protozoa contributed $31.9 \%$ with 340 individuals from eight (8) taxa, Copepods, on the other hand, had seven (7) taxa and 223 individuals contributing $20.9 \%$ of the total population. The least was Cladocerans with five (5) taxa and 147 individuals contributing 13.8\% of the population. Spatially, the highest number of individuals was recorded in station 1 (388) representing $36.4 \%$ of zooplankton, station 2 accounted for 303 individuals, with a relative abundance of $28.4 \%$, station 3 had 375 individual and a relative abundance of $35.2 \%$. There was no significant difference $(P>0.05)$ in 
Table 3. The Spatial Zooplankton Composition, Abundance and Distribution

\begin{tabular}{|c|c|c|c|c|c|}
\hline $\begin{array}{c}\text { Taxonomic } \\
\text { Group }\end{array}$ & Species Composition & Stn. 1 & Stn. 2 & Stn. 3 & Total \\
\hline \multirow[t]{8}{*}{ Protozoa } & Carchesium polypium & 2 & 4 & 0 & 6 \\
\hline & Didinium bolbanni & 9 & 28 & 20 & 57 \\
\hline & Tintinnopsis lacustries & 18 & 10 & 0 & 28 \\
\hline & Paramecium caudatum & 3 & 8 & 12 & 23 \\
\hline & Oikomonas rostratum & 0 & 0 & 35 & 35 \\
\hline & Spirostomum ambiguum & 35 & 28 & 19 & 82 \\
\hline & S. teres & 28 & 10 & 17 & 55 \\
\hline & S. macronucleus & 19 & 24 & 11 & 54 \\
\hline \multirow[t]{5}{*}{ Cladocera } & Alona exima & 8 & 0 & 16 & 24 \\
\hline & Alona rectangular & 4 & 0 & 21 & 25 \\
\hline & Bosmina longirotris & 9 & 13 & 3 & 25 \\
\hline & Chydorus piger & 20 & 35 & 0 & 55 \\
\hline & Pleuroxus levis & 0 & 0 & 18 & 18 \\
\hline \multirow[t]{10}{*}{ Rotifera } & Lecane bulla & 27 & 0 & 32 & 59 \\
\hline & L. quadridentata & 26 & 0 & 19 & 45 \\
\hline & L. langsenensis & 14 & 3 & 14 & 31 \\
\hline & Asplanchna priodonata & 17 & 31 & 0 & 48 \\
\hline & Keratella cochlearis & 0 & 0 & 16 & 16 \\
\hline & K. quarata & 0 & 0 & 13 & 13 \\
\hline & Asplanchna brightwelli & 10 & 26 & 19 & 55 \\
\hline & Gastropus hyptopus & 8 & 0 & 0 & 8 \\
\hline & Synchaeta pectinata & 2 & 10 & 8 & 20 \\
\hline & Conochilus unicornis & 28 & 14 & 19 & 61 \\
\hline \multirow[t]{7}{*}{ Copepoda } & Calanus glacialis & 14 & 0 & 6 & 20 \\
\hline & Cyclops strenuous & 16 & 24 & 18 & 58 \\
\hline & Diaphanasoma excisum & 7 & 0 & 0 & 7 \\
\hline & Tropocyclops prasinus & 24 & 19 & 29 & 72 \\
\hline & Eucalanus elongates & 14 & 3 & 10 & 27 \\
\hline & Nauplius & 18 & 0 & 0 & 18 \\
\hline & Zoeo larva & 8 & 13 & 0 & 21 \\
\hline
\end{tabular}

the number of individuals recorded in the stations when ANOVA was applied. Temporally, the highest number of individuals (193) was recorded in August 2019 and the lowest (50) in December 2019. August 2019 was significantly different $(P<0.05)$ from September, November, and December 2019 when ANOVA was applied.

The community structure as shown by biodiversity indices are presented in Tables 5 and 6 . The spatial diversity indices showed that Shannon-Wiener index $(\mathrm{H})$ was highest in station 1 (3.069) and least (2.703) in station 2
(Table 4). Margalef's index (d) was also highest in station 1(4.194) and the least in station 2 (2.975). Evenness index value was different with the highest recorded in station 3 (0.9029) and least $(0.8279)$ in station 1 . The temporal diversity indices showed that Shannon-Wiener index $(\mathrm{H})$ was highest in January $2020(2.808)$ and least (2.063) in September 2019 (Table 5). Margalef's index (d) was also highest in January 2020 (4.747) and the least in September 2019 (2.172). Evenness index value was also different from the highest recorded in July (0.8269) and least (0.6991) in November 2019. 
Table 4. The Temporal Zooplankton Composition, Abundance and Distribution

\begin{tabular}{|c|c|c|c|c|c|c|c|c|c|c|c|}
\hline \multirow[b]{2}{*}{$\begin{array}{c}\text { Taxonomic } \\
\text { Group }\end{array}$} & \multirow[b]{2}{*}{ Species (Taxa) } & \multirow[b]{2}{*}{ May } & \multirow[b]{2}{*}{ June } & \multicolumn{3}{|c|}{2019} & \multirow[b]{2}{*}{ Oct. } & \multirow[b]{2}{*}{ Nov. } & \multirow[b]{2}{*}{ Dec. } & \multicolumn{2}{|c|}{2020} \\
\hline & & & & July & Aug. & Sept. & & & & Jan. & Feb. \\
\hline \multirow[t]{8}{*}{ Protozoa } & Carchesium polypium & 0 & 1 & 1 & 1 & 2 & 0 & 0 & 1 & 0 & 0 \\
\hline & Didinium bolbanii & 10 & 10 & 13 & 0 & 4 & 0 & 8 & 8 & 0 & 4 \\
\hline & Tintinnopsis lacustries & 2 & 0 & 4 & 6 & 1 & 4 & 2 & 7 & 1 & 1 \\
\hline & Paramecium caudatum & 4 & 0 & 0 & 4 & 4 & 1 & 0 & 0 & 10 & 0 \\
\hline & Oikomonas rostratum & 8 & 3 & 4 & 0 & 3 & 4 & 0 & 2 & 3 & 8 \\
\hline & Spirostomum ambiguum & 0 & 5 & 15 & 25 & 8 & 0 & 18 & 8 & 3 & 0 \\
\hline & S. teres & 8 & 16 & 0 & 14 & 0 & 2 & 10 & 3 & 2 & 0 \\
\hline & S. macronucleus & 0 & 15 & 14 & 3 & 8 & 9 & 0 & 3 & 2 & 0 \\
\hline \multirow[t]{5}{*}{ Cladocera } & Alona exima & 3 & 0 & 10 & 11 & 0 & 0 & 0 & 0 & 0 & 0 \\
\hline & Alona rectangular & 4 & 0 & 8 & 3 & 0 & 0 & 2 & 1 & 1 & 6 \\
\hline & Bosmina longirotris & 10 & 0 & 5 & 0 & 5 & 1 & 0 & 2 & 2 & 0 \\
\hline & Chydorus piger & 10 & 0 & 2 & 20 & 13 & 6 & 0 & 0 & 4 & 0 \\
\hline & Pleuroxus levis & 0 & 0 & 0 & 0 & 0 & 8 & 0 & 2 & 4 & 4 \\
\hline \multirow[t]{10}{*}{ Rotifera } & Lecane bulla & 0 & 22 & 18 & 0 & 0 & 0 & 7 & 0 & 3 & 9 \\
\hline & L. quadridentata & 3 & 0 & 18 & 12 & 0 & 0 & 4 & 0 & 8 & 0 \\
\hline & L. langsenensis & 0 & 10 & 0 & 6 & 0 & 8 & 0 & 0 & 3 & 4 \\
\hline & Asplanchria priodonata & 0 & 2 & 5 & 3 & 15 & 5 & 8 & 0 & 5 & 5 \\
\hline & Keratella cochlearis & 1 & 22 & 18 & 10 & 0 & 0 & 0 & 2 & 2 & 0 \\
\hline & K. quaratqa & 2 & 10 & 0 & 3 & 0 & 0 & 0 & 1 & 0 & 0 \\
\hline & Asplanchina brihtwelli & 0 & 0 & 9 & 1 & 0 & 0 & 2 & 0 & 1 & 0 \\
\hline & Gastropus hyptopus & 2 & 3 & 0 & 1 & 0 & 0 & 0 & 0 & 2 & 0 \\
\hline & Sychaeta pectinata & 1 & 1 & 6 & 0 & 0 & 3 & 2 & 0 & 0 & 7 \\
\hline & Conochilus unicornis & 0 & 10 & 15 & 18 & 0 & 0 & 0 & 4 & 8 & 6 \\
\hline \multirow[t]{7}{*}{ Copepoda } & Calanus glacialis & 0 & 0 & 0 & 5 & 0 & 4 & 1 & 0 & 5 & 5 \\
\hline & Cyclops strenuous & 10 & 0 & 0 & 8 & 0 & 13 & 0 & 0 & 10 & 17 \\
\hline & Diaphanasoma excisum & 0 & 0 & 0 & 0 & 0 & 0 & 3 & 3 & 0 & 1 \\
\hline & Tropocyclops prasinus & 15 & 0 & 0 & 18 & 0 & 20 & 0 & 0 & 19 & 0 \\
\hline & Eucalanus elongates & 10 & 0 & 0 & 7 & 0 & 0 & 0 & 0 & 4 & 6 \\
\hline & Nauplius $s p$ & 0 & 0 & 0 & 14 & 0 & 0 & 2 & 0 & 0 & 2 \\
\hline & Zoeo larva & 10 & 0 & 0 & 0 & 0 & 6 & 1 & 3 & 1 & 1 \\
\hline
\end{tabular}

Table 5. The Spatial Diversity indices of Zooplankton Community

\begin{tabular}{lccc}
\hline Ecological indices & STN. 1 & STN. 2 & STN. 3 \\
\hline No. of species & 26 & 18 & 22 \\
No. of individual & 388 & 303 & 375 \\
Margalef's index (D) & 4.194 & 2.975 & 3.543 \\
Shannon-wiener (H) & 3.069 & 2.703 & 2.989 \\
Evenness (E) & 0.8279 & 0.8293 & 0.9029 \\
\hline
\end{tabular}

Table 6. The Temporal Diversity indices of Zooplankton Community

\begin{tabular}{lllllllllll}
\hline $\begin{array}{l}\text { Ecological } \\
\text { Indices }\end{array}$ & $\begin{array}{l}\mathbf{2 0 1 9} \\
\text { May }\end{array}$ & Jun & Jul & Aug. & Sep & Oct. & Nov. & Dec. & $\begin{array}{l}\text { Ja20 } \\
\text { Jan. }\end{array}$ & Feb. \\
\hline No. of Species & 18 & 14 & 17 & 22 & 10 & 15 & 14 & 15 & 23 & 16 \\
No. of Individuals & 113 & 130 & 165 & 193 & 63 & 94 & 70 & 50 & 103 & 86 \\
Margalef Index (D) & 3.596 & 2.671 & 3.134 & 3.990 & 2.172 & 3.081 & 3.060 & 3.579 & 4.747 & 3.367 \\
Shannon-wiener (H) & 2.658 & 2.336 & 2.643 & 2.796 & 2.063 & 2.446 & 2.281 & 2.489 & 2.808 & 2.541 \\
Evenness (E) & 0.792 & 0.739 & 0.827 & 0.744 & 0.787 & 0.769 & 0.699 & 0.803 & 0.721 & 0.793 \\
\hline
\end{tabular}


3. 3. Relationship between the zooplankton groups and environmental variables

The canonical correspondence analysis (CCA) ordination showed a good relationship between the zooplankton groups and environmental variables. The strongest explanatory factors spatially were Dissolved oxygen (DO), temperature, and hydrogen ion $(\mathrm{pH})$. DO was a major factor in station 1 while temperature, and $\mathrm{pH}$ were major factors in station 2. The strongest negative influences were from phosphate, BOD, nitrate, and turbidity. The CCA triplot also revealed that DO exerted a greater positive influence on Copepod group abundance while electrical conductivity negatively influenced the abundance of the rotifer group, and BOD, nitrate, and turbidity negatively influenced the protozoa group (Figure 2).

On the other hand, the strongest explanatory factors temporally were $\mathrm{pH}$ and temperature. The $\mathrm{pH}$ was a major factor in January and February 2020 while the temperature was a major factor in October 2019. The strongest negative influences were from phosphate and nitrate. The CCA triplot also revealed that temperature exerted a greater positive influence on Copepod group abundance in October 2019 while BOD negatively influenced the abundance of the rotifer group in June and July 2019, and nitrate influenced the protozoa group in November 2019 (Figure 3).

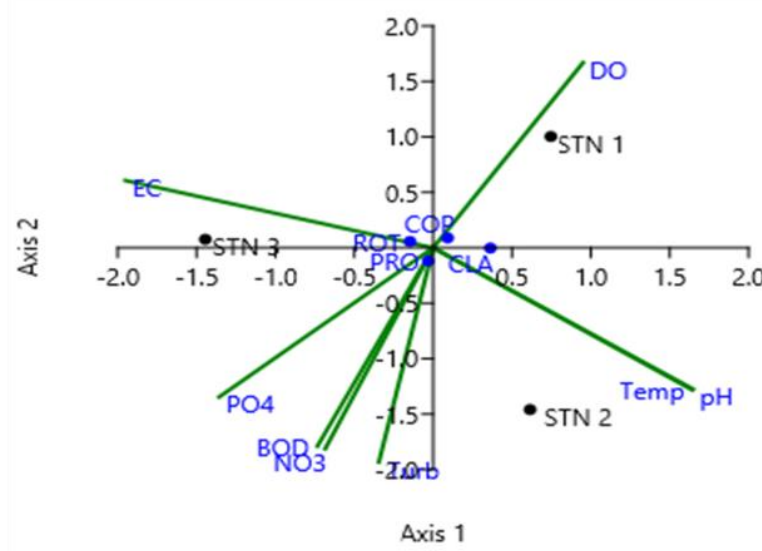

Figure 2. Canonical correspondence analysis (CCA) ordination showing relationships between zooplankton groups, stations and environmental variables. (BOD - biochemical oxygen demand, DO - dissolved oxygen, Temp - water temperature, $\mathrm{NO}_{3}$ - nitrates, $\mathrm{PO}_{4}$ - phosphates, EC- electrical conductivity, Turb - turbidity, PRO - protozoa, ROT - rotifer, CLA - cladocera and COP - copepod)

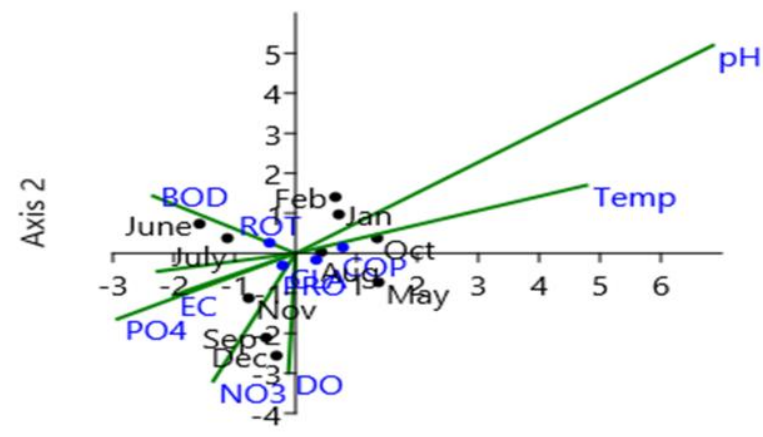

Axis 1

Figure 3. Canonical correspondence analysis (CCA) ordination showing relationships between zooplankton groups, months and environmental variables. (BOD - biochemical oxygen demand, DO - dissolved oxygen, Temp - water temperature, $\mathrm{NO}_{3}$ - nitrates, $\mathrm{PO}_{4}$ - phosphates, EC- electrical conductivity, Turb - turbidity, PRO - protozoa, ROT - rotifer, CLA - cladocera, COP - copepod, Jan - January, Feb - February, Aug August, Sep - September, Oct - October, Nov - November and Dec - December) 


\section{Discussion}

The water quality of any given water body is influenced by physical, chemical, and anthropogenic factors. Water temperature values were within the same range recorded by Akpan \& Etim (2015) in Uta Ewa Estuary. The variations in the spatial and temporal water temperatures could be attributed to variability in weather conditions and time of sampling. Higher temperatures were recorded between December 2019 and February 2020, which was the peak of the dry season. In the tropics, the wet season results in lower water temperatures while the dry season brings about higher temperatures (Anyanwu, 2012; Etesin et al., 2013; Houssou et al., 2017). Idowu et al. (2013) reported that the higher water temperatures recorded during the dry season could be as a result of low precipitation, high atmospheric temperature and low relative humidity. A similar trend was observed by George \& Atakpa (2015) in Cross River Estuary and Ukpatu et al. (2018) in Okoro River estuary, southeast Nigeria. Surface water temperature is a necessary ecological factor that controls chemical and physiological processes as well as the distribution of aquatic organisms (Ukpatu et al., 2018).

Higher conductivity was recorded in station 3 ; this could be attributed to tidal action which enhances the seawater intrusion towards the station by the river mouth (Gasim et al., 2015). This is in line with Kaniz et al. (2014) that recorded the highest conductivity in station 6 by the mouth of the river. Higher values were also recorded in May and September 2019 in station 3 which could be attributed to anthropogenic activities exacerbated by season and tidal effect. Kaniz et al. (2014) also recorded high values during the wet season. High electrical conductivity values have been associated with sand mining activities, and dredging (Ohimain et al., 2008; Seiyaboh et al., 2013; Rehman et al., 2016; Akankali et al., 2017). Conductivity values of between 25 and $100 \mathrm{mS} / \mathrm{m}$ are characteristic of water bodies rich in electrolytes and are categorized as eutrophic (Bellos \& Sawidis 2005). The $\mathrm{pH}$ values were moderate acidic to alkaline spatially and temporally. The values were within the range recorded by Gasim et al. (2015) in Paka River, Kuala Terengganu, Malaysia, and Ukpatu et al. (2018) in Okoro River Estuary, South East, Nigeria. The highest $\mathrm{pH}$ value was recorded in October 2019 in station 2. High $\mathrm{pH}$ values recorded were ascribed to the buffering capacity of seawater (Anila-Kumary et al., 2007; Desai et al., 2020).
Higher values were also recorded in January and February 2020 which could be attributed to high photosynthetic activity (Ukpatu et al., 2018).

Turbidity values were high in stations 2 and 3; this could be traceable to dumping of allochthonous materials into the water body at these stations. Jonah et al. (2019) and Anyanwu \& Umeham (2020a) reported that high turbidity is associated with indiscriminate dumping of refuse, and other human activities. The high values recorded from June to September 2019 could be as a result of increased precipitation transporting allochthonous materials into the water body.

Dissolved oxygen (DO) is an important parameter that determined the survival of some aquatic organisms. Lower values were recorded in stations 2 and 3 attributed to the constant release of wastes generated from the nearby settlements, and local markets into the water. Ayobahan et al. (2014) reported that reduction in dissolved oxygen levels is attributed to the presence of organic pollutants in the water resulting from human activities. The depletion of dissolved oxygen occurs during the degradation of organic matters by bacteria in the water (Mahre et al., 2007; Ukpatu et al., 2018). Relatively higher DO was recorded in station 1 which could be attributed to low human activities. Kaniz et al. (2014) and Hastuti et al. (2018) also recorded the highest DO in the upstream station (station 1). Temporally, the DO value increased with the rains with the lowest mean value recorded during the August break 2019. Ukpatu et al. (2018) also recorded higher DO values during the wet season in Okoro River Estuary, South East, Nigeria and attributed it to the significant influence of freshwater input during the wet season

Biochemical Oxygen Demand had an inverse trend of the dissolved oxygen. Relatively higher values were recorded in stations 2 and 3; attribute to anthropogenic activities. BOD is an indicator of organic pollution in a water body, and affects water quality (Nwankwo et al., 2014). The BOD values were higher during the wet season between May and September 2019 attributable to the biodegradation of allochthonous input from flash floods. This trend and value range was reported by Ukpatu et al. (2018) in Okoro River Estuary, South East, Nigeria.

Phosphate values followed the same trend as BOD. Higher values were recorded in stations 2 and 3; which could be attributed to 
anthropogenic activities in the stations. The main source of phosphate in the estuarine and coastal waters is the seawater (Liu et al., 2009) except for those receiving freshwater contaminated with wastes containing detergents and phosphate-phosphorous fertilizers and pesticides from agricultural areas (Ajithkumar et al., 2006; Mandal et al., 2012). Concentration in the freshwater that mixes with the seawater determined the concentration of phosphate in coastal water (Paytan and Mclaughlin, 2007). High mean values were recorded during the wet season between May and September 2019; attributable to the decomposable organic matter brought in by surface runoffs and high anthropogenic activities during this period. The observed trend was in line with George \& Efiom (2018) and Jonah et al. (2020). The phosphate values were relatively higher than values recorded by Kaniz et al. (2014) in Merbok Estuary, Kedah, Malaysia.

Nitrate exhibited the same spatial trend with $\mathrm{BOD}$ and phosphate; significantly higher values were recorded in stations 2 and 3 attributed to the anthropogenic activities in the stations. High mean values were also recorded during the wet season between May and September 2019 as in phosphate; attributable to the decomposable organic matter brought in by surface runoffs and high anthropogenic activities during this period as well as the atmospheric deposition (USEPA, 2010). Selvam et al. (1994) reported that an increase in nitrate values in mangrove waters was caused by the decomposition of organic matter. The nitrate values were also relatively higher than values recorded by Kaniz et al. (2014) in Merbok Estuary, Kedah, Malaysia.

Water quality characteristics have an enormous impact on the growth and abundance of zooplankton fauna (Suresh et al., 2011; Anyanwu et al., 2013; Essien-lbok \& Ekpo 2015; Jonah \& George 2019). Alexander (2012) affirmed that the occurrence of planktonic fauna depends on certain factors such as climate change, habit structure in terms of physicochemical properties, and biotic factors. This study revealed that environmental variability, season, and anthropogenic perturbation influenced the species composition, abundance and distribution. The 30 species (taxa) recorded was low compared with 66 taxa reported by Ekwu and Sikoki (2005) in lower Cross River Estuary; 44 species reported by Eyo et al. (2013) but higher than 9 taxa reported by Obot et al. (2020) in a tropical coastal creek,
Southeastern Nigeria. The low taxa recorded could be attributed to effect of anthropogenic activities in the estuary exacerbated by season. Xiong et al. (2016) observed that the consequences of direct and/or indirect pollution arising from human activities usually result in low biodiversity of aquatic organisms. The total number of individuals recorded in this study was far lower than the lowest value $(3,381$ individuals) recorded in Zone III of Abdul et al. (2016). The Zooplankton species were dominated by Rotifera. This is in line with Imoobe \& Akoma (2009), Imoobe \& Adeyinka (2010), and Abdul et al. (2016). The dominance of rotifera was attributed to selective predation pressure from planktivorous fishes on bigger sized zooplankton and their reproductive success enhanced by short developmental rates when the conditions are favorable (Imoobe \& Adeyinka 2009; Abdul et al., 2016; Obot et al., 2020). The highest abundance was recorded in station 1 which could be ascribed to favorable conditions; station 1 receives more freshwater input and less perturbed. This is in line with Arimoro \& Oganah (2010) and Arimoro et al. (2018). The temporal abundance increased with the rains from May to August 2019; this could be attributed to high precipitation, low temperatures and food availability which favored their development. The highest abundance was recorded in August 2019 when the region usually experiences a break in wet season precipitation (August break). During the break, the water mass is stable because of low hydrodynamics, shallow depths and high nutrient input (Marques et al. 2002) resulting in higher zooplankton abundance. Related studies have reported high zooplankton abundance during the wet season months; ascribed to favorable conditions especially increased nutrients (phosphate and nitrate) and food availability in the water body brought in via surface run-off (Adesalu et al., 2010; Balogun \& Ladigbolu 2010; Ekpo 2013; Essien-lbok \& Ekpo 2015).

Zooplankton community structure showed that the water quality of Uta Ewa Estuary has not been adversely impacted by anthropogenic activities and season. Olawusi-Peters and Ajibare (2014) reported that comparison of communities to identify biotic disturbances or level of stability can be done with species diversity indices as useful tools while Leinster and Cobbold (2012) reported the indices increases as the complexity or stability of the habitat increases. The Shannon-Weiner diversity value recorded in station 1 (3.069) was 
of the range indicating a stable environment while values recorded in stations 2 (2.703) and 3 (2.989) and temporally (2.063 - 2.808) indicated mild pollution. The values were relatively higher than the spatial values recorded by Ajuonu et al. (2011) in Bonny Estuary, Nigeria but lower than the values recorded by Abdul et al. (2016) in the tropical coastal estuary, South-west, Nigeria. This observation could be attributed to the effect of anthropogenic activities exacerbated by season. Akindele and Olutona (2014) recorded Shannon-Weiner indices values of $<3.0$, which was attributed to polluted and unstable environment. Bonny Estuary is the home to the Nigeria Liquified Natural Gas Project, which could seriously impact on the environment. The Shannon-weiner diversity index usually ranges between 1.5 and 3.5 ; rarely exceeding 4.5 (Magurran, 1988; Bibi and Ali, 2013). The index categories indicated that values of $<1$ is for heavily polluted conditions, values of 1 to 2 is for moderate polluted conditions, and values of $>3$ for stable environmental conditions (Stub et al., 1970; Mason, 2002). The Margalef indices were high for stations 1 (4.194) and 3 (3.543) indicating some level of stability. The values were higher than spatial values recorded by Ajuonu et al. (2011) in Bonny Estuary, Nigeria, and Abdul et al. (2016) in a tropical coastal estuary, South-west, Nigeria. Station 2 (2.975) had value that is higher than Ajuonu et al. (2011) But lower than Abdul et al. (2016) attributable to the effect observed in ShannonWeiner indices. Akindele and Olutona (2014) also recorded Margalef indices values of between 1 and 3 ; which also attributed to environmental pollution and instability. Margalef index has no upper limit but most of the diversity indices give high values to stable communities and low values to unstable ones; thereby revealing environment variability attributed to environmental degradation (Mason 2002; Yeom and Kim, 2011; Shah and Pandit, 2013). The pielou's evenness values were high, closer to one (1) in all the stations $(0.8279-0.9029)$ especially in station $3(0.9029)$ and months (0.699 - 0.827); indicating that there is no dominance of any particular species or group of species. The spatial values were higher than the station values recorded by Abdul et al. (2016) in the tropical coastal estuary, South-west, Nigeria. According to Leinster and Cobbold (2012), evenness is an important aspect of diversity indices showing how evenly distributed the individuals are within the different species.
Canonical Corresponding Analysis (CCA) revealed that the zooplankton group variation patterns were significantly related to the environmental variation patterns of the estuary (Abdul et al., 2016; Sharif et al., 2017). The eight environmental parameters significantly either positively or negatively influenced the variations in the composition of the zooplankton community. Each of the zooplankton groups has the ecological adaptations to thrive in an environment where their survival and reproduction are optimal (Rougier et al., 2005). DO and temperature strongly influenced the copepods spatially and temporally, respectively while electrical conductivity negatively influenced the rotifer group and the protozoa group was negatively influenced by BOD, nitrate, and turbidity in the stations. On the other hand, BOD had a negative influence on the rotifer group and nitrate on the protozoa group during the sampling period. Temperature plays an important role in determining the seasonal variation in zooplankton species composition (David et al., 2005; Lionard et al., 2005). The Zooplankton community is structured most importantly by changes in environmental factors like $\mathrm{pH}$, phosphorus, dissolved oxygen, conductivity, and water temperature (Arimoro et al., 2018).

\section{Conclusion}

This study showed that the water quality and zooplankton community was influenced by anthropogenic activities both spatially and temporally but the season also played a major role in the temporal variation. The biodiversity indices pointed to a slightly polluted to the stable environment. In conclusion, the water quality was deteriorating due to anthropogenic activities, which in turn affected the structure of the zooplankton community

\section{References}

Abdul, W.O.,Adekoya, E.O., Ademolu, K.O., Omoniyi, I. T., Odulate, D. O., Akindokum, T. E., Olajide, A. E. 2016. The effects of environmental parameters on zooplankton assemblages in tropical coastal estuary, south-west, Nigeria. Egyptian Journal of Aquatic research.

Adesalu,T., Bagbe, M., Keyede, D. 2010. Hydrochemistry and phytoplankton Composition of two Tidal Creeks in SouthWestern Nigeria. International Journal of Tropical Biology 58(3): 827-840. 
Ajithkumar, T. T.,Thangaradjou, T., Kannan, L. 2006. Physico-chemical and biological properties of the Muthupettai mangrove in Tamil Nadu. Journal of the Marine Biological Association of India 48:131-138.

Ajuonu, N., Ukaonu, S.U., Oluwajoba, E.O., Mbawuike, B.E., Williams, A.B., Myade, E. F. 2011. The abundance and distribution of plankton species in the bonny estuary; Nigeria. Agriculture and Biology Journal of North America 6:1032-1037. Doi:10.5251/abjna.2011.2.6.1032.1037

Akankali, J.A., Idongesit, A.S., Akpan, P.E. 2017. Effects of sand mining activities on water quality of Okoro Nsit stream, Nsit Atai Local Government Area, Akwa Ibom State, Nigeria. International Journal of Development and Sustainability 6:451462.

Akindele, E.O., Olutona, G.O. 2014. Water Physicochemistry and Zooplankton Fauna of Aiba Reservoir Headwater Streams, Iwo, Nigeria. Journal of Ecosystems, Article ID 105405.

Akpan, A.U., Egwali, E.C, Esenowo I.K., Akpabio, E.K. 2019. Preliminary study on the composition and abundance of shellfish (Decapoda: Crustacea) in Uta Ewa Estuary, Ikot Abasi Local Government Area, Akwa Ibom State, Nigeria. Animal Research International 16(1): 3198-3206.

Akpan, I.I., Etim. U. 2015. Observations on the variations (seasonal and spatial) in water quality and ecological adversity of Uta Ewa. Niger Delta Region, Nigeria. International Journal of Oceanography and Marine Ecological System 4 (1): 31-35.

Alexander, R. 2012. Interactions of zooplankton and phytoplankton with cyanobacteria. M.Sc. Dissertation, University of Nebraska, Dissertations \& Theses in Natural Resources. 58.

Anila-Kumary, K.S.A., Azis, P.K.A., Natarajan, P. 2007. Water quality of the Adimalathura estuary, Southwest coast of India. Journal of the Marine Biological Association of India 49(1):1-6.

Anitha, G., Kumar, S.P. 2013. Seasonal variations in physico-chemical parameters of Thengapattanam estuary, Southwest coastal zone, Tamil Nadu, India. International Journal of Environmental Sciences 3(4):1253-1261.
Anyanwu, E.D. 2012. Physicochemical and Some Trace of Metal Analysis of Ogba River, Benin City, Nigeria. Jordan Journal of Biological Science 5:47-54.

Anyanwu, E.D., Umeham, S.N. 2020a. Identification of waterbody status in Nigeria using predictive index assessment tools: a case study of Eme River, Umuahia, Nigeria.International Journal of Energy and Water Resources.

Anyanwu, E.D., Umeham, S.N. 2020b. An index approach to heavy metal pollution assessment of Eme River, Umuahia, Nigeria. Sustainability, Agri, Food and Environmental Research, 8(X), 2020. doi.org/10.7770/safer-VONO-art2067.

Anyanwu, E.D., Ikomi, R.B., Arimoro F.O. 2013. Water quality and zooplankton of the Ogba River, Benin City, Nigeria. African Journal of Aquatic Science 38(2):193-199.

AOAC 2000.Official Methods of Analysis, Association of Official Analytical Chemist (15th Edition),Washington DC: Academic Publishers 480p.

APHA 2005.Standard Methods for the Examination of Water and Wastewater. American Public Health Association (21st Edition). Washington DC.

Arimoro, F.O., Oganah, A.O. 2010. Zooplankton community responses in a perturbed tropical stream in Niger Delta, Nigeria. The Open Environmental and Biological Monitoring Journal 3:1-11.

Arimoro, F.O., Odume, O.H., Uhunoma, S.I., Edegbene, A.A. 2015. Anthropogenic Impact on Water Chemistry and Benthic Macroinvertebrate Associated Changes in a Southern Nigerian Stream. Environmental Monitoring and Assessment, 187: 14.

Arimoro, F.O, Olisa, H.E., Keke, U.N., Ayanwale, A.V., Chukwuemeka, V.I., 2018. Exploring spatio-temporal patterns of plankton diversity and community structure as correlates of water quality in a tropical stream. Acta Ecologica Sinica 38: 216-223.

Ayobahan, S.U., Ezenwu, I.M., Orogun, E.E., Uriri, J.E., Wemimo, I.J. 2014. Assessment of Anthropogenic Activities on Water Quality of Benin River. Journal of Applied Science and Environmental Management 18(4):629-636. 
Balogun, K., Ladigbolu, I.A. 2010. Nutrients and phytoplankton production dynamics of a tropical harbor in Relation to Water Quality Indices. Journal of American Science 6(9): 261-275.

Bellos, D., Sawidis, T.2005. Chemical pollution monitoring of the River Pinios (ThessaliaGreece). Journal of Environmental Management 76(4): 282-292.

Bibi, F., Ali, Z. 2013. Measurement of diversity indices of avian communities at Taunsa barrage wildlife sanctuary, Pakistan. Journal of Animal and Plant Science 23(2): 469-474.

Boxshall, G.A, Braide, E.I. 1991. The freshwater cyclopoid copepods of Nigeria, with an illustrated key to all species. Bulletin of British Museum of Natural History (Zool.) 57: 185-212.

Dang, P.D., Khoi, N.V., Nga, L.N., Thanh, D.N., Hai, H.T. 2015. Identification Handbook of Freshwater Zooplankton of the Mekong River and its Tributaries, Mekong River Commission, Vientiane. 207pp.

David, V., Sautour, B., Chardy, P., Laconte, M. 2005. Long-term changes of the zooplankton variability in a turbid environment: The Gironde estuary (France). Estuarine, Coastal and Shelf Science 64, 171-184.

Davis, O.A., Ugwumba, A.A.A. 2013. Tidal influence on nutrient status and phytoplankton population of Okpoka Creek, Upper Bonny Estuary, Nigeria. Journal of Marine Biology, 1-16. Doi.org/10.1155/2013/684739.

Desai, N.C., Kukadiya, N.B., Mehta, J.P., Godhani, D.R., Lakhmapurkar, J., Dave, B.P. 2020. Elucidation of tidal spatialtemporal variation of physico-chemical and nutrient parameters of estuarine water at South Gujarat. World Scientific News 143:79-102

Dorak, Z. 2013. Zooplankton Abundance in the Lower Sakarya River Basin (Turkey): Impact of Environmental Variables. Journal of Black sea/Mediterranean Environment 19(1): 1-22.

Egwali, E.C., Akpan, A.U., Esenowo, I.K., Akpabio, E.K. 2018. The Composition and Abundance of Shellfish (Decapoda: Crustacea) in Uta Ewa Estuary, Ikot Abasi Local Government Area, Akwa lbom State,
Nigeria. Proceedings of 6th NSCB Biodiversity Conference (Uniuyo 2018), University of Uyo, Nigeria. $125-130$.

Ekpo, I. 2013. Effect of physico-chemical parameters on zooplankton species density of a Tropical Rainforest River in Niger Delta, Nigeria using Conical Cluster Analysis. International Journal of Engineering and Science 2(4): 13-21.

Ekwu, O., Sikoki, F.D. 2005. Species composition and distribution of zooplankton in the Lower Cross River Estuary. African Journal of Applied Zoology and Environmental Biology 7: 510.

Ergönül, M.B., Erdoğan, S., Altindağ, A., Atasağun, S. 2016. Rotifera and Cladocera fauna of several lakes from the Central Anatolia, Marmara, and Western Black Sea regions of Turkey. Turkish Journal of Zoology 40: 141-146.

Esenowo, I.K., Akpan, A.U., Egwali, E.C., Akpabio, E.E. 2016. The Abundance and Composition of Crabs (Decapoda) in Uta Ewa Brackish Water, Akwa Ibom State, South-South, Nigeria. Journal of Applied Sciences and Environmental Management 20(4): 919-924.

Essien-lbok, M., Ekpo I. 2015. Physicochemical factors influencing zooplankton community structure of a Tropical River, Niger Delta, Nigeria. Journal of Environmental and Earth Science 5(17): 162-173.

Etesin, U., Udoinyang, E., Harry, T. 2013. Seasonal Variation of Physicochemical Parameters of Water and Sediments from Iko River, Nigeria. Journal of Environmental and Earth Science 3: 96110.

Eyo, V.O., Andem, A. B., Ekpo, P. B. 2013. Ecology and diversity of zooplankton in the Great Kwa River, Cross River State, Nigeria. International Journal of Science and Research, 2(10): 67-71.

Ferdous, Z., Muktadir, A.K.M. 2009. A Review: Potentiality of zooplankton of bioindicators. American Journal of Applied Science 6(10): 1815-1819.

Gasim, M.B., Khalid, N.A., Muhamad, H. 2015. The Influence of Tidal Activities on Water Quality of Paka River Terengganu, 
Malaysia. Malaysian Journal of Analytical Sciences 19(5): 979 - 990

George, U.U., Efiom, E. 2018. Physical and chemical variations in water quality of Imo River owing to Human Perturbations in the system. Researcher 10(6): 47-54.

George, U. U, Atakpa, E.O. 2015. Seasonal variation in physico-chemical characteristics of Cross River Estuary, South Eastern Nigeria. Nature and Science 13(12): 86-93.

Hammer,O., Harper, D.A.T., Ryan, P.D. 2001. PAST: paleontological statistics Software Package for Education and Data Analysis. Paleontologia Electronica, 4,9.

Hastuti, A.W., Pancawati, Y., Surana, I.N. 2018. The abundance and spatial distribution of plankton communities in Perancak Estuary, Bali. IOP Conf. Series: Earth and Environmental Science, 176: 012042. Doi:10.1088/1755-1315/176/1/012042

Houssou, A.M., Ahouansou-Montcho, S., Montchowui, E., Bonou, C.A. 2017. Spatial and Seasonal Characterization of Water Quality in the Ouémé River Basin (Republic of Benin, West Africa). Egyptian Journal of Chemistry 60: 1077-1090.

Idowu, E.O., Ugwumba, A.A.A., Edward, J.B., Oso, J.A. 2013. Study of the Seasonal Variation in the Physico-Chemical Parameters of a Tropical Reservoir. Greener Journal of Physical Sciences 3(4): 142-148.

Imoobe, T.O.T, Adeyinka, M.L. 2010. Zooplankton-based assessment of the trophic state of a tropical forest river. International Journal Fisheries and Aquaculture 2(2): 64-70.

Imoobe, T.O.T., Akoma, O.C. 2009. Spatial variations in the composition and abundance of zooplankton in the Bahir Dar Gulf of Lake Tana, Ethiopia. African Journal of Ecology 48: 72-77.

Jeje. C.Y.1988. A revision of the Nigerian species of the genera Mesocyclops sars, 1914 and Thermocyclops kiefer, 1927 (Copepoda; Cyclopoida). Hydrobiologia 164: 171-184

Jeje, C.Y., Fernando, C.H. 1986. A practical guide to the identification of Nigerian Zooplankton (Cladocera, Copepoda and Rotifera). Published by Kainji Lake Research Institute, Kainji, Nigeria.142pp.
Job, B.E., Ekpo, P.J., Asuquo, P.E. 2017. Impact of human activities on the distribution, abundance and diversity of zooplankton in the Calabar River system, Cross River State, Nigeria, International Journal of Science information 2(7): 412425.

Jonah, U.E., George, U.U. 2019. Influence of water quality on zooplankton community of Etim Ekpo River, Akwa Ibom State, Nigeria. World Rural Observation 11 (3): 49-57.

Jonah, U.E.,George ,U.U., Avoaja, D.A. 2020. Impacts of agrochemical on water quality and macroinvertebrates abundance and distribution in Ikpe Ikot Nkon River, SouthSouth, Nigeria. Researcher 12(1): 36-43.

Jonah, U.E., George, U.U., Avoaja, D.A. 2019. Impacts of anthropogenic perturbation on water quality characteristics of Ikpe Ikot Nkon River, Southern Nigeria. New York Science Journal 12(9): 70-77.

Kaniz, F., Wan, M.W.O, Mansor, M.I. 2014. Spatial and Temporal Variation of Physicochemical Parameters in the Merbok Estuary, Kedah, Malaysia. Tropical Life Sciences Research, 25(2): 1-19.

Kar S, Das P, Das U, Bimola M, Kar D, Aditya G. 2018. Correspondence of zooplankton assemblage and water quality in wetlands of Cachar, Assam, India: Implications for environmental management. Limnological Review 18(1): 9-19

Kutama RM, Abubakar MM, Balorabe ML. 2014. The plankton as indicators of water quality in Kusalla Reserviour: A Shallow manmade Lake. IOSR Journal of Pharmacy and Biological Sciences 9 (3): 12-15.

Leinster T, Cobbold CA. 2012.Measuring diversity: the importance of species similarity. Ecology 93(3): 477-489.

Lin Q, You WH, Xu FJ. Yu QJ, Yu HG. 2014. Zooplankton community structure and its relationship with environmental factors in Dishui Lake. Acta Ecologica Sinica 34: 6918-6929.

Lionard M, Aze'mar F, Boule^treau S, Muylaert K, Tackx M, Vyverman W. 2005. Grazing by meso- and microzooplankton on phytoplankton in the upper reaches of the Schelde estuary (Belgium/ The 
Netherlands). Estuarine, Coastal and Shelf Science 64: 764-774.

Liu SM, Hong GH, Ye XW, Zang J, Jiang XL. 2009. Nutrient budgets for large Chinese estuaries and embayment. Biogeosciences 6:391-435.

Magurran AE. 1988. Ecological Diversity and its Measurement. Croom Helm, London.

Mahre MY, Akan JC, Moses EA, Ogugbuaja VO. 2007. Pollution indicators in River Kaduna, Kaduna State, Nigeria, Trends in Applied Sciences Research 2: $304-311$.

Mandal SH, Das A, Nanda AK. 2012. Study of some physico-chemical water quality parameters of Karola River, West Bengal: An attempt to estimate pollution status. International Journal of Environmental Protection 2 (8): 16-22.

Marques JC, Graca, MA, Pardal MA. 2002. Introducing the Mondego River Basin. In Pardal, M.A., Marques, J.C. and Graca, M. A. (eds). Aquatic Ecology of the Mondego River Basin. Global Importance of Local Experience. Imprensa da Universidade, Coimbra, p. 7-12.

Mason, CF. 2002. Biology of Fresh Water Pollution. (4th Edition). Pearson Educational Limited, Essex, U.K.

Morris, A.W., Allen, J.I., Howland, R., Wood, R.G. 1995. The estuary plume zone: Source or sink for land derived nutrient discharges? Estuarine, Coastal and Shelf Science 40(4): 387-402.

Nwankwo, C., Mohammed, A., Ikyereve, E.R., Dawari, B.K. 2014. The Impact of human Water Exploitation of Physicochemical characteristics of Mmubete River in the Niger Delta, Nigeria. International Journal of Science and Technology 3:292-297.

Obot, O.I., David, G.S., Ekpo, I.E. 2020. Zooplankton Assemblages of a tropical coastal creek, Southeastern Nigeria. Ecologia 10: 63 - 70 .

Ohimain, E.I., Imoobe, T.O., Bawo, D.S. 2008. Changes in Water Physico-Chemical Properties Following the Dredging of an Oil Well Access Canal in the Niger Delta. World Journal of Agricultural Science 4: 752-758.

Olawusi-Peters, O.O., Ajibare, A.O. 2014. Species richness, diversity and abundance of some Decapod Crustaceans in coastal waters of Ondo State, South West, Nigeria. International Journal of Fauna and Biological Studies 1: 44-51.

Paytan, A., Mclaughlin, K. 2007. The oceanic phosphorus cycle. Chemical Reviews, 107, 563-576.

Rehman, M., Yousuf, A.R., Balkhi, M.H., Rather, M.I., Shahi, N., Meraj, M., Hassan, K. 2016. Dredging induced changes in zooplankton community and water quality in Dal Lake, Kashmir, India. African Journal of Environmental Science and Technology 10: 141-149.

Roman, M.R., Pierson, J.J., Kimmel, D.G., Boicourt, W.C., Zhang, X. 2012. Impacts of hypoxia on zooplankton spatial distributions in the Northern Gulf of Mexico. Estuaries and Coasts 35: 1261-1269.

Rougier, C., Pourriot, R., Lam-Hoai T, Guiral, D. 2005. Ecological patterns of the rotifer communities in the Kaw River estuary (French Guiana). Estuarine, Coastal and Shelf Science 63: 83-91.

Schoen, S. (1988). Cell counting. In: Experimental: A Laboratory Manual (Lobban, C.S., Chapman, D.J. and Kremer, B.P. (Eds.). Cambridge University Press, Cambridge.

Seiyaboh, E., Ogamba, E.N. Utibe, D. I. 2013. Impact of Dredging on the water quality of Igbedi Creek, Upper Nun River, Niger Delta, Nigeria. IOSR Journal of Environmental Science, Toxicolology and Food Technology 7: 51-56.

Selvam, V.,Hariprasad, V., Mohan, R., Ramasubramanian, R. 1994. Diurnal variations in the water quality of sewage polluted Adayar mangrove water, east coast of India. Indian Journal of Marine Sciences 23: 94-97.

Shah, J.A., Pandit, A.K. 2013. Application of diversity indices to crustacean community of Wular Lake, Kashmir Himalaya. International Journal of Biodiversity and Conservation, 5(6): 311-316.

Sharif, A.S.M., Islam, M., Bhuyan, S. 2017. Zooplankton in the lower Meghna River and its Estuary with Relation to PhysicoChemical Parameters, Bangladesh. International Journal of Zoology Studies 2(2): 06-15 
Sharma, S., Siddique, A., Singh., K., Chouhan, M., Vyas, A., Solnki., C.M, Sharma, D., Nair, S., Sengupta, T. 2010: Population dynamics and seasonal abundance of zooplankton community in Narmada River (India). Researcher 2:1-9.

Sousa, W., Attayde, J. L, da Silva-Rocha, E.,Eskinazi-Sant'Anna, E.M. 2008. The response of zooplankton assemblages to variations in the water quality of four manmade lakes in semi-arid Northeastern Brazil. Journal of Plankton Research 30: 699-708.

Stub, R., Appling, J.W., Hatstetter, A.M., Hass, I.J. 1970. The effect of industrial waste of Memphis and Shelby country on primary planktonic producers. Bioscience 20: 905912.

Suresh, S.,Thirumala, S., Ravind, H. 2011. Zooplankton diversity and its relationship with physico-chemical parameters in Kundavada Lake of Davangere District, Karnataka, India. Pro-Environment 4:5659.

Ukpatu. J., Udoinyang, E., Etim, L. 2018. Seasonality, Collinearity and Quality Assessment of the Physicochemical
Properties of Okoro River Estuary, South Eastern Nigeria. Journal of Scientific Research and Reports 19(5):1-15.

USEPA 2010. Nutrients in Estuaries. A Summary Report of the National Estuarine Experts Workgroup 2005-2007.United States Environmental Protection Agency. https://www.epa.gov/sites/production/files/ documents/nutrients-in-estuariesnovember-2010.pdf

Vincent, K., Mwebaza-Ndawula, L., Makanga, B., Nachuha, S. 2012. Variations in zooplankton community structure and water quality conditions in three habitat types in northern Lake Victoria. - Lakes \& Reservoirs: Research and Managment 17: 83-95.

Xiong, W., Li, J., Chen, Y., Shan, B., Wang, W., Zhan, A. 2016. Determinants of community structure of zooplankton in heavily polluted river ecosystems. Scientific Reports 6, 22043: 1 - 11. Doi: 10.1038/srep22043.

Yeom, D.J., Kim, J.H. 2011. Comparative evaluation of species diversity indices in the natural deciduous forest of Mt. Jeombong. Forrest Science and Technology 7: 68-74 